\title{
A CMOS Four-Quadrant Analog Current Multiplier
}

\author{
Remco J. Wiegerink \\ MESA Research Institute \\ Twente University \\ P.O. Box 217 , \\ 7500 AE Enschede, The Netherlands. \\ phone: $x-3153892736$ \\ fax: $x-3153354003$
}

\begin{abstract}
In this paper a CMOS four-quadrant amalog current multiplier is deseribed. The cireuit is based on the square-law charneteristic of an MOS transistor and is inseastive to temperature and process variations. Furthermore the circult is boensitive to the body effect so it is not necessary to place transistars in individual wells. The multiplier hos a lare $3 \mathrm{~dB}$ bandwith $(69 \mathrm{MHz}$ with $10 \mathrm{~m}$ transisters) and an approxdmately constant imput impedance. The circult was realized on a CMOS semleustom array. Measurements have shown that the non-linearity is less than $1 \%$ at the maximua input current range and less than $0.2 \%$ when the inpent range is restricted to s0\% of the mendinm.
\end{abstract}

\section{INTRODUCTION}

The quarter square principle is a well known technique to realize a multiplier. Various multipliers based on this principle have been reported [1], [2], [3]. For signals in the form of currents the principle can be written as:

$$
I_{o}=\left(I_{x}+I_{y}\right)^{2}-\left(I_{x}-I_{y}\right)^{2}=4 I_{x} I_{y}
$$

So, in order to multiply the input currents $I_{x}$ and $I_{y}$ we can square both the sum and the difference of these currents and then subtract the results from eachother.

In the next section a suitable current squarer will be described. In section III, two of these squaring circuits will be combined to realize the four-quadrant current multiplier. Section IV describes second-order effects. The results of simulations and measurements on both the squaring circuit and the complete multiplier are presented in section $\mathrm{V}$.

\section{The CuRrent-Squaring Circuit}

A schematic diagram of the squaring circuit is shown in fig. 1. All transistors are equal and operate in the saturation region. Using a simple square-law model, the drain current of an MOS transistor in the saturation region can be written as:

$$
I_{d}=k\left(V_{g s}-V_{t}\right)^{2}, \text { with } k=\frac{1}{2} \mu C_{a x} \frac{W}{L}
$$

Or:

$$
V_{g s}=V_{t}+\sqrt{I_{d} / k}
$$

The transistors $\mathbf{M}_{1}, \mathbf{M}_{2}, \mathrm{M}_{3}$ and $\mathrm{M}_{4}$ are connected in an
MOS translinear loop [4]. From Kirchhoff's voltage law, it follows that:

$$
V_{851}+V_{g \circ 2}=V_{g \times 3}+V_{g \times 4}
$$

Substituting eqn. (3) in eqn. (4) results in:

$$
\begin{aligned}
& V_{t 1}+\sqrt{\frac{I_{1}}{k_{1}}}+V_{t 2}+\sqrt{\frac{I_{2}}{k_{2}}}= \\
& V_{t 3}+\sqrt{\frac{I_{3}}{k_{3}}}+V_{t 4}+\sqrt{\frac{I_{4}}{k_{4}}}
\end{aligned}
$$

If the body-effect is represented as a change in threshold voltage as a function of the source-bulk voltage it is easily seen that the effect is equal for transistors $M_{1}$ and $M_{4}$ and transistors $\mathrm{M}_{2}$ and $\mathrm{M}_{3}$. Therefore, $V_{t 1}$ will be equal to $V_{t 4}$ and $V_{R 2}$ will be equal to $V_{B}$ and the threshold voltages can be dropped from eqn. (5). If we assume equal transistors and neglect the slight influence of the body-effect on $k_{1}, k_{2}$, $k_{3}$ and $k_{4}$, these factors can also be dropped. The result is:

$$
\sqrt{I_{1}}+\sqrt{I_{2}}=\sqrt{I_{3}}+\sqrt{I_{4}}
$$

This relation is temperature and process independent.

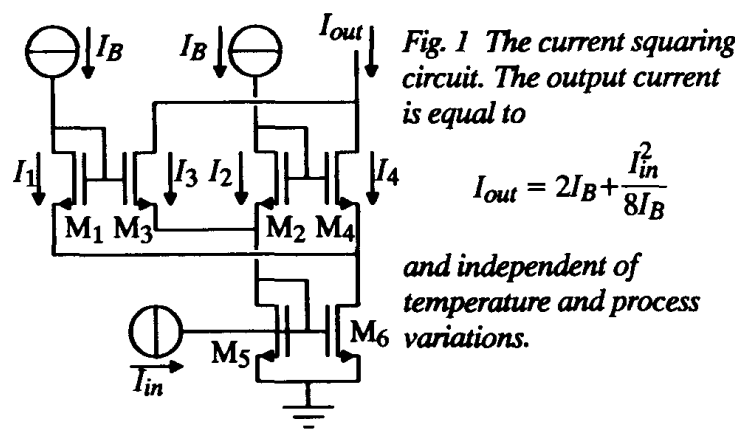

The currents $I_{1}$ and $I_{2}$ are each forced equal to $I_{B}$. Therefore eqn. (6) becomes:

$$
\sqrt{I_{3}}+\sqrt{T_{4}}=\sqrt{4 I_{B}}
$$

From (7) we can obtain an expression for the current $I_{3}+I_{4}$, which is the output current of the circuit: 


$$
\begin{aligned}
& \sqrt{I_{3}}+\sqrt{I_{4}}=\sqrt{4 I_{B}} \\
& \rightarrow I_{3}+I_{4}+2 \sqrt{I_{3} I_{4}}=4 I_{B} \\
& \rightarrow\left(I_{3}+I_{4}-4 I_{B}\right)^{2}=4 I_{3} I_{4} \\
& \rightarrow I_{3}^{2}+2 I_{3} I_{4}+I_{4}^{2}-8 I_{B}\left(I_{3}+I_{4}\right)+16 I_{B}^{2}=4 I_{3} I_{4} \\
& \rightarrow\left(I_{3}-I_{4}\right)^{2}+16 I_{B}^{2}=8 I_{B}\left(I_{3}+I_{4}\right) \\
& \rightarrow I_{3}+I_{4}=2 I_{B}+\frac{\left(I_{3}-I_{4}\right)^{2}}{8 I_{B}}=I_{\text {out }}
\end{aligned}
$$

The current mirror $\mathbf{M}_{5}, \mathbf{M}_{6}$ forces the relation:

$$
I_{\text {in }}+I_{2}+I_{3}=I_{1}+I_{4}
$$

$I_{1}$ and $I_{2}$ are equal to $I_{B}$ and can be dropped from eqn. (9). Therefore

$$
I_{\text {in }}=I_{4}-I_{3}
$$

Substituting eqn. (10) in eqn. (8) results in

$$
I_{\text {out }}=2 I_{B}+\frac{I_{\text {in }}^{2}}{8 I_{B}}
$$

However, this relation is only valid if all transistors operate in the saturation region. This imposes some restrictions on the input current range. If $I_{\text {in }}=0$, the draincurrent of transistors $\mathrm{M}_{1}, \mathrm{M}_{2}, \mathrm{M}_{3}$ and $\mathrm{M}_{4}$ will be equal to $I_{B}$. If $I_{\text {in }}$ increases in either direction, the current through transistors $M_{1}$ and $M_{2}$ will remain constant but the current through either $\mathrm{M}_{3}$ or $\mathrm{M}_{4}$ will increase while the other decreases. The maximum input current is reached when $I_{3}$ or $I_{4}$ becomes zero. From equations $(8)$ and $(10)$ the following expressions for $I_{3}$ and $I_{4}$ can be derived:

$$
I_{3}=I_{B}-\frac{I_{\text {in }}}{2}+\frac{I_{\text {in }}^{2}}{16 I_{B}} \quad, \quad I_{4}=I_{B}+\frac{I_{\text {in }}}{2}+\frac{I_{\text {in }}^{2}}{16 I_{B}}
$$

These currents are both larger than or equal to zero if the input current is restricted to:

$$
-4 I_{B} \leq I_{\text {in }} \leq 4 I_{B}
$$

The current through the current mirror $\mathrm{M}_{5}, \mathrm{M}_{6}$ is equal to $I_{1}+I_{4}$ and is therefore larger than $I_{4}$ and does not add an extra restriction to the range of $I_{\text {in }}$. Outside the interval specified by (14) the output current increases linear with In .

It can be shown that the value of the input resistance $R_{\text {in }}$ of the circuit is dependent of the input current $I_{i n}$. The circuit can be improved by adding two current sources with value $I_{B}$ at both sides of the current mirror as illustrated by fig. 2 . In that case the current through the current mirror becomes equal to the current through transistor $\mathbf{M}_{4}$. Substituting the expression for this current (eqn. (12)) in eqn. (3) gives:

$$
V_{g s, \mathrm{M}_{5}}=V_{t}+\sqrt{\frac{I_{B}}{k_{5}}}+\sqrt{\frac{1}{16 I_{B} k_{5}}} \cdot I_{\text {in }}
$$

So, the value of $R_{\text {in }}$ becomes independent of $I_{\text {in }}$ :

$$
R_{\text {in }}=\sqrt{\frac{1}{16 I_{B} k_{5}}}
$$
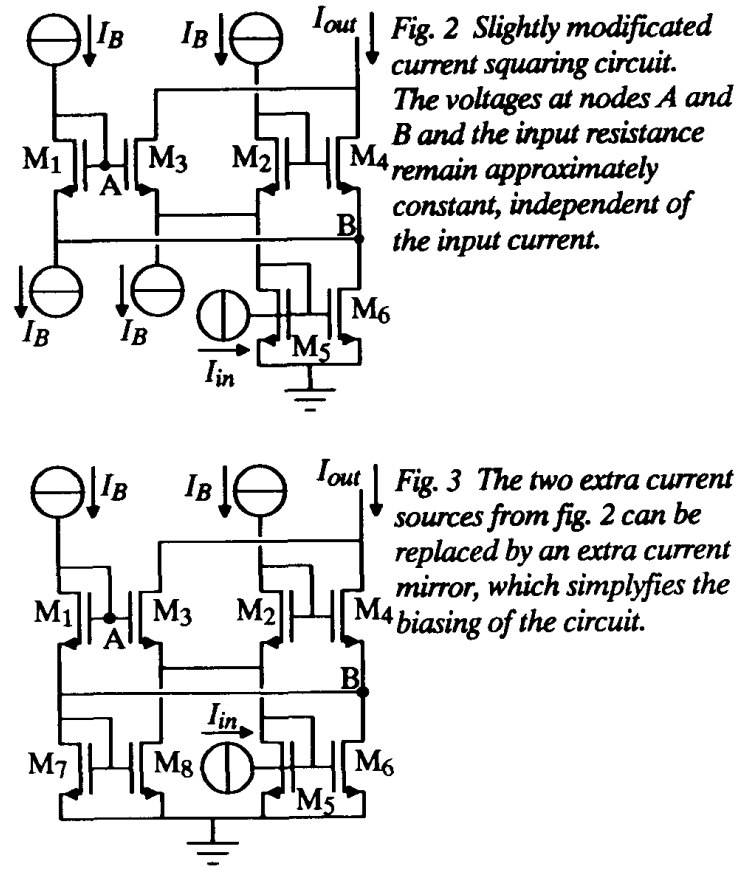

The fact that the current through the current mirror equals the current through transistor $\mathbf{M}_{4}$ implies further that apart from an offset due to the body-effect the gate-source voltage of $\mathrm{M}_{5}$ equals the gate-source voltage of $\mathrm{M}_{4}$. Therefore, the sum of the gate-source voltages of $\mathrm{M}_{5}$ and $M_{3}$ will be constant and independent of the input current. This means that the voltages at nodes $A$ and $B$ in fig. 2 will be constant, which is favourable for the high-frequency behaviour of the circuit.

The fact that the voltage at node $B$ remains constant implies that the two extra current sources needed in fig. 2 can be replaced by an extra current mirror as shown by fig. 3. The circuit now becomes fully symmetrical and node $B$ can also be used as a second input. The effective input current is equal to the normal input current minus the input current at node $B$.

\section{THE FOUR-QUADRANT CURRENT MULTIPLIER}

Fig. 4 shows how we can realize a complete four-quadrant current multiplier using two current squarers. The input currents of the squaring circuits are $\left(I_{y}-I_{x}\right)$ and $\left(I_{y}+I_{x}\right)$. It

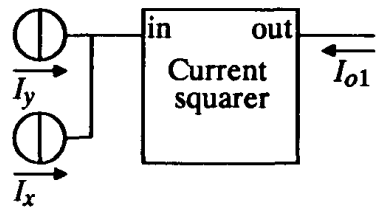

Fig. 4 The complete four quadrant current multiplier consists of two current squaring circuits. The output current is equal to:

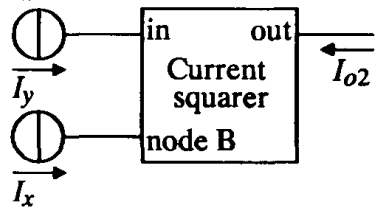


follows from eqn. (11) that the output currents $I_{o 1}$ and $I_{o 2}$ will be equal to:

$$
\begin{aligned}
& I_{o 1}=2 I_{B}+\frac{\left(I_{y}+I_{x}\right)^{2}}{8 I_{B}} \\
& I_{o 2}=2 I_{B}+\frac{\left(I_{y}-I_{x}\right)^{2}}{8 I_{B}}
\end{aligned}
$$

So, the difference $I_{\text {out }}$ will be:

$$
I_{o u t}=I_{o 1}-I_{o 2}=\frac{I_{x} I_{y}}{2 I_{B}}
$$

The input current range of the multiplier follows from (13) and is equal to:

$$
\left|I_{x}\right|+\left|I_{y}\right| \leq 4 I_{B}
$$

\section{SECOND-ORDER EFFECTS}

In the previous sections second-order effects like mobility reduction, channel length modulation and component mismatch were neglected. These effects will be considered in this section.

\section{A. Mobility Reduction}

The mobility reduction in an MOS transistor can be modeled as follows:

$$
I_{d}=\frac{k}{\left(1+\theta\left(V_{g}-V_{t}\right)\right)} \cdot\left(V_{g s}-V_{t}\right)^{2}
$$

The mobility reduction parameter $\theta$ is process dependent and has typical values between 0.01 and $0.2 \mathrm{~V}^{1}$. If we incorporate the mobility reduction effect, eqn. (6) for the current squaring circuit becomes:

$$
\begin{gathered}
\sqrt{I_{1} \cdot\left(1+\theta\left(V_{g s 1}-V_{t}\right)\right)}+\sqrt{I_{2} \cdot\left(1+\theta\left(V_{g s 2}-V_{t}\right)\right)}= \\
\sqrt{I_{3} \cdot\left(1+\theta\left(V_{g s}-V_{t}\right)\right)}+\sqrt{I_{4} \cdot\left(1+\theta\left(V_{g S 4}-V_{t}\right)\right)}
\end{gathered}
$$

Using (21) instead of (6) we can find the following approximation for the output current of the current squaring circuit:

$$
\begin{gathered}
I_{\text {out }} \approx 2 I_{B}+\frac{I_{\text {in }}^{2}}{8 I_{B}} \cdot\left(1-\Theta+\frac{\Theta^{2}}{4}\right) \\
+\frac{I_{\text {in }}^{4}}{2 \cdot\left(4 I_{B}\right)^{3}} \cdot \theta \cdot\left(1+\frac{\Theta}{2}\right) \\
+\frac{I_{\text {in }}^{6}}{8 \cdot\left(4 I_{B}\right)^{5}} \cdot \Theta^{2}
\end{gathered}
$$

with:

$$
\begin{aligned}
& \Theta=\frac{\theta\left(V_{g s B}-V_{t}\right)}{1+\theta\left(V_{g s}-V_{t}\right)} \\
& V_{g S B}=V_{g S 1}=V_{g s 2}
\end{aligned}
$$

If we compare eqn. (22) with the ideal response (11), we see that the size of the quadratic component $I_{\text {in }}^{2}$ is slightly reduced and that a fourth and a sixth order term are introduced. For practical values of $\theta=0.035 \mathrm{~V}^{1}$ and
$\left(V_{g s B}-V_{t}\right)=1.0 \mathrm{~V}$ the calculated maximum deviation from the ideal output characteristic (11) is approx. $0.4 \%$ of the maximum output current. This error increases approx. linear with $\theta$. The error caused by mobility reduction can be reduced by duplicating the circuit and eliminating the fourth order term [5].

\section{B. Channel length Modulation}

In the current squaring circuits channel length modulation could cause an error in the transfer of the current mirror $M_{5}, M_{6}$ due to the difference between the drain-source voltages of $M_{5}$ and $M_{6}$. If the voltage at the output of the circuit varies, channel length modulation could also occur in transistors $\mathrm{M}_{3}$ and $\mathrm{M}_{4}$ If short channels are used it might be neccessary to cascode the output of the circuit.

\section{Component Mismatches}

Another possible source of errors is component mismatch. In the current squaring circuits component mismatches could result in slightly different values for the current sources $I_{B}$. The result will be an input offset current.

If a mismatch in the threshold voltages of transistors $\mathrm{M}_{1}$.. $\mathrm{M}_{4}$ occurs, these voltages can not be dropped from eqn. (5). It can be shown that such a threshold mismatch has the same effect as an error in the value of $I_{B}$.

The most serious errors occur from a mismatch between transistors $\mathbf{M}_{3}$ and $\mathbf{M}_{4}$ or an error in the current mirror $\mathbf{M}_{5}$ $M_{6}$. These mismatches result in an output current that contains terms linear with $I_{i n}$ and $I_{\text {in }}{ }^{3}$.

\section{SimULATEd AND MEASUREd ReSUlts}

SPICE simulations were performed for both the current squarer and the complete multiplier circuit. The simulations showed that a $-3 \mathrm{~dB}$ bandwidth of $50 \mathrm{MHz}$ is feasible using transistors with a channel length of $10 \mathrm{um}$. This corresponds to the following approximation that can be derived for the $-\mathbf{3 d B}$ frequency:

$$
\omega_{-3 \mathrm{~dB}} \approx \frac{g_{m, I_{\mathrm{B}}}}{\sqrt{2} \cdot C_{8 s}}
$$

Where $g_{m, I_{\mathrm{B}}}$ is the transconductance of a transistor with drain current $I_{B}$ and $C_{g s}$ is the gate source capacitance of the transistors.

The circuits have been realized on the ACMA semicustom chip fabricated in the UT CMOS process. All transistors had a channel length of $10 \mu \mathrm{m}$ and a channel width of $50 \mu \mathrm{m}$. Fig. 4 shows the measured output current of the current squaring circuits and its first derivative. The response is the same for all three versions of the circuit. The value of the bias current $I_{B}$ was $100 \mu \mathrm{A}$. It can be seen that the square-law behaviour is very good. For input currents outside the range specified by (13) the output current increases linear with the input current as expected.

Fig. 5 shows the measured input voltage and input resistance of the three current squaring circuits. As expected, the input resistance of the circuit from fig. 1 is dependent of the input current. The improved circuit from fig. 2 has a constant input resistance over a wide range of 


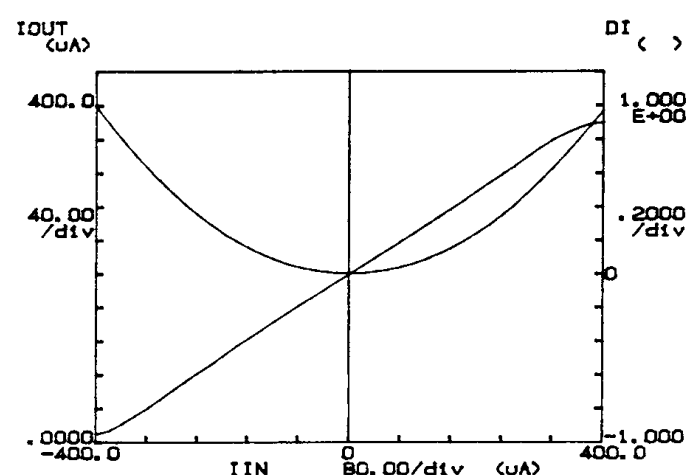

Fig. 5 Measured output current of the current squaring circuits and its first derivative.

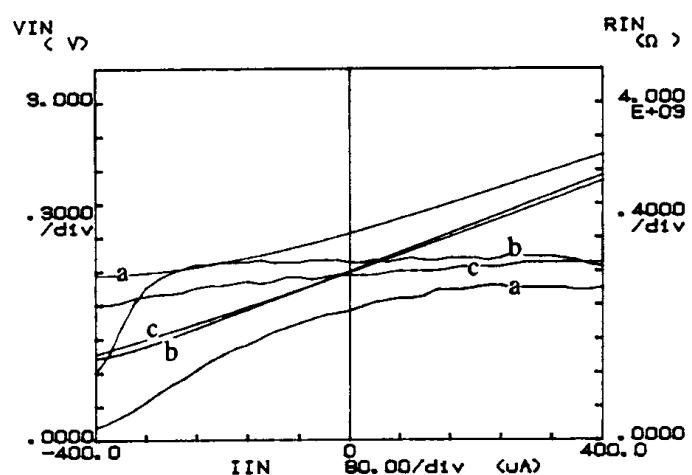

Fig. 6 Measured input voltage and input resistance of the current squaring circuits from (a) fig. 1, (b) fig. 2 and (c) fig. 3 .

the input current but it decreases for large negative values of $I_{\text {in }}$. The input resistance of the circuit from fig. 3 only varies slightly with $I_{\text {in }}$.

The response of the complete multiplier circuit has also been measured. Fig. 6 shows a plot of the output current as a function of one of the input currents $\left(I_{x}\right)$ while the other input current $\left(I_{y}\right)$ remains fixed. From the derivative DI it can be seen that the multiplier has a very good linearity. If the non-linearity NL is defined as

$$
\mathrm{NL}=\frac{\text { max. deviation from a linear response }}{\text { max. output current }} \cdot 100 \%,
$$

the non-linearity is less than $1 \%$ over the entire input current range specified by (19). If the input currents are restricted to $50 \%$ of their maximum value, the nonlinearity becomes less than $0.2 \%$.

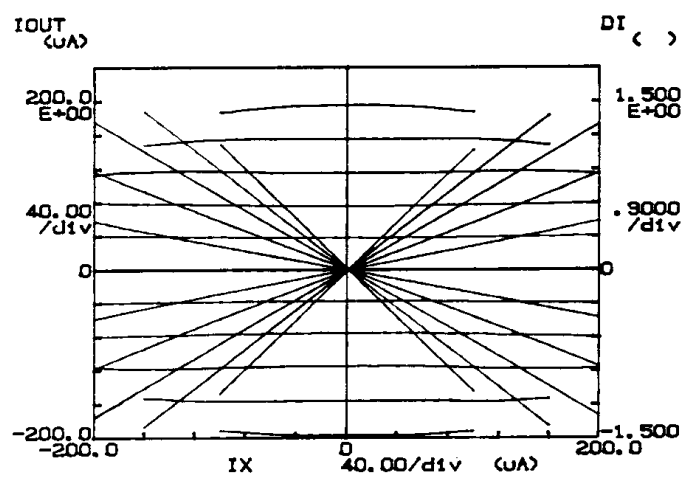

Fig. 6 Measured response of a complete four-quadrant current multiplier as a function of input current $I_{x}$ The other input current (Iy) varies from $-300 \mu \mathrm{A}$ to $300 \mu \mathrm{A}$ in steps of $60 \mu \mathrm{A}$. The first derivative is also shown.

\section{CONCLUSION}

A new CMOS four-quadrant current multiplier has been presented. The circuit features a large $-3 \mathrm{~dB}$ bandwidth, a good linearity and an approximately constant input impedance and is insensitive to temperature and process variations. Furthermore the circuit does not suffer from the body effect so it is not neccessary to place transistors in individual wells.

\section{Acknowledgements}

The author wishes to thank E. Seevinck, R.F. Wassenaar and E. Klumperink for fruitful discussions and F. van Dam and J. van Sinderen for performing the SPICE simulations and breadboard measurements.

\section{References}

[1] K. Bult and H. Wallinga, "A class of analog CMOS circuits based on the square-law characteristic of an MOS transistor in saturation," IEEE J. Solid-State Cincuits, Vol. SC-22, pp. 357-365, June 1987.

[2] J.S. Pena-Finol and J.A. Connelly, "A MOS four-quadrant analog multiplier using the quarter-square technique," IEEE J. Solid-State Circuits, Vol. SC-22, pp. 1064-1073, Dec. 1987.

[3] H. Song and C. Kim, "An MOS four-quadrant analog multiplier using simple two-input squaring circuits with source followers," IEEE J. Solid-State Circuits, Vol. SC-25, pp. 841-847, June 1990.

[4] E. Seevinck and R.J. Wiegerink, "Generalized translinear circuit principle," accepted for publication in the IEEE J. Solid-State Circuits.

[5] A. Cichocki and R. Unbehauen, "Technique for compensation of errors in analogue multipliers," Electronics Letters, Vol. 25, pp. 305-307, March 1989. 